ArtefaCToS. Revista de estudios de la ciencia y la tecnología

eISSN: $1989-3612$

Vol. 8, No. 2 (2019), 2. ${ }^{a}$ Época, 125-137

DOI: http://dx.doi.org/10.14201/art201982125137

\title{
Hilary Putnam: un alegato a favor de la objetividad
}

\author{
Hilary Putnam: an apology to objectivity
}

\section{Laura Balvanera DI SANTO}

Universidad Nacional de Tucumán/CONICET, Argentina lauradisanto.89@gmail.com

Recibido: 06/07/2019. Revisado: 11/09/2019. Aceptado: 26/09/2019

\section{Resumen}

El propósito de este trabajo es analizar y evaluar la defensa de Putnam del status normativo y objetivo del pensamiento ético. Luego, pretendo mostrar que de ella se desprende una noción diferente de objetividad, que implica una idea de racionalidad más amplia que la científica, que reconoce la pluralidad de puntos de vista y que está comprometida con una reflexión inteligente de nuestras aserciones. A estos fines, examinaré el problema de la objetividad en las obras de Putnam desde diferentes ángulos, en primer lugar, el rechazo a los supuestos en los que se asienta la dicotomía hecho-valor, en segundo lugar, los supuestos epistemológicos del positivismo lógico y su postura acerca de los juicios de valor, y en tercer lugar, desde el análisis de la noción misma de objetividad y su relación con las prácticas lingüísticas.

Palabras claves: Hilary Putnam; ética; objetividad; justificación; racionalidad.

\begin{abstract}
The aim of this work is to survey Putnam's defense of objectivity and normativity in the ethic's realm. I pretend to show an alternative conception of objectivity which involves a broader aproach to racionality than the scientific. Moreover, this approach acknowledges pluralities of points of view and it's committed to reflective thinking about our assertions. Then, I will examine the question of objectivity in Putnam's papers, according to different outlooks: firstly, Putnam's rejection to the traditional fact-value dichotomy, secondly, the Logical Postivism' claim and his attitude about values judgments, finally, from the survey of the term objectivity and his relationship with linguistic practices.
\end{abstract}

Keywords: Hilary Putnam; ethics; objectivity; justification; rationality. 


\section{Introducción}

Hilary Putnam dedicó a lo largo de su vida un considerable esfuerzo intelectual por defender que hay mejores y peores perspectivas, mejores y peores interpretaciones, estilos de vida o modos de comportamiento. Sostuvo incansablemente que podemos afirmarlo tanto en las ciencias como en la ética y que podemos hablar de objetividad en tales áreas del conocimiento y de la experiencia humana. Esto lo llevó a preocuparse por la controvertida cuestión acerca de cómo es posible que las afirmaciones valorativas sean objetivas y a dedicarse a leer en detalle a los pragmatistas americanos para dar cuerpo a sus respuestas. Este interés fue constante a lo largo de sus obras, en las cuales discutió con representantes de tendencias relativistas, con filósofos que guiados por una concepción de la racionalidad y la objetividad absoluta relegaron a las cuestiones de la ética y de la vida humana al plano de lo puramente subjetivo y con aquellos que proponen enfoques ontológicos de la misma.

En las obras de su madurez, Putnam deja entrever una concepción personal acerca de la ética influenciada por el pragmatismo americano, comprendida como un complejo de preocupaciones interrelacionadas, que abarcan desde la afirmación de principios abstractos, como los Derechos Humanos, hasta la solución de problemas prácticos y contextualizados. Esta visión no restringe la incumbencia de esta disciplina a un sólo objeto o interés sino, por el contrario, pretende hacer lugar a diferentes inquietudes que aquejan a los hombres en cada momento histórico.

El autor considera que la idea de que los juicios de valor son subjetivos es una creación filosófica, que ha sido aceptada por mucha gente como si fuera de sentido común y desarrollada de distintas maneras. Por tal motivo, el problema de la objetividad de los juicios de valor puede analizarse en sus obras desde diferentes ángulos: 1) el rechazo a los supuestos en los que se asienta la dicotomía hecho-valor, 2) los supuestos epistemológicos del positivismo lógico y su postura acerca de los juicios de valor, 3) el análisis de la noción misma de objetividad y su relación con las prácticas lingüísticas, o sea, un enfoque pragmalingüístico, entre otros. En este trabajo me propongo analizar y evaluar la defensa putneana del status normativo y objetivo del pensamiento ético a partir de tales enfoques. Luego, pretendo mostrar que de ella se desprende una noción de objetividad alternativa, que implica una idea de racionalidad más amplia que la científica, que reconoce la pluralidad de puntos de vista y que está comprometida con una reflexión inteligente de las valoraciones.

\section{La dicotomía hecho-valor y la objetividad}

En "La ciencia y algunos filósofos" el autor discute contra aquellos pensadores que en base a una dicotomía tajante entre hechos y valores mantienen la tesis de 
que los enunciados fácticos pueden ser objetivamente verdaderos y objetivamente justificados mientras que los juicios de valor no. Añade al respecto:

Los positivistas lógicos afirmaron haber mostrado que las proposiciones éticas sólo parecen ser aserciones legítimas, pues, realmente, carecen de valor de verdad, $\mathrm{y}$, de hecho, están por completo fuera de la esfera de la argumentación racional. Es obvio (...) que no creyeron que una afirmación de este tipo constituyera en sí misma una metafísica. Por lo contrario, prefirieron pensar que al proscribir la ética de la esfera de las cuestiones racionalmente discutibles, habían hecho "un descubrimiento lógico". Y, desafortunadamente, en la actualidad multitud de personas que nunca han escuchado de una posición llamada "positivismo lógico" hablan como si fuese evidente de por sí que las posiciones éticas son esencialmente subjetivas. (Putnam, 2007, 173)

Esta idea de que los juicios de valor están completamente al margen de la esfera de la razón la propone el joven Wittgenstein, quien en la Conferencia sobre ética expresa que hablar sobre ética es arremeter contra los límites del lenguaje, ya que éste no puede expresar valores absolutos. En la medida que surge del deseo de decir algo sobre el sentido de la vida, sobre lo absolutamente bueno o valioso no puede ser una ciencia, no acrecienta en nada a nuestro conocimiento (Wittgenstein, 1989, 43).

En El desplome de la dicotomía hecho-valor el autor elabora una reconstrucción histórica de tal dicotomía, que va desde Hume hasta el positivismo lógico. En ella muestra que ciertas distinciones filosóficas, como el caso de la distinción entre los hechos y los valores, se han exagerado por los filósofos hasta alcanzar el rango de dicotomía. El primer antecedente de tal "dicotomía" es la distinción de Hume entre relaciones de ideas y cuestiones de hecho. Luego, en el siglo $\mathrm{XX}$, los positivistas lógicos mantuvieron una separación tajante entre hechos y valores, y formularon un criterio de significatividad cognitiva según el cual entre los enunciados "científicamente significativos" sólo hay enunciados analíticos y enunciados sintéticos. Éstos obstaculizaron nuestro pensamiento tanto en lo que concierne al razonamiento ético como a la descripción del mundo, según Putnam, impidiéndonos ver que la evaluación y la descripción están entretejidas y son interdependientes $(2004,16)$.

La idea de que los hechos y los valores están imbricados en toda área del discurso, conocida como "tesis de la imbricación", proviene de los pragmatistas clásicos, quienes sostuvieron que el valor y la normatividad permean la totalidad de la experiencia. Si observamos el vocabulario de nuestro lenguaje en su totalidad y no la pequeña porción que los positivistas lógicos consideran suficiente para la descripción de los "hechos", dice Putnam, nos encontraremos con una imbricación mucho más profunda de hechos y valores, que incluye valores de distintos tipos $(2002,49)$. Para mostrar esta imbricación una de las maniobras argumentativas utilizadas consiste en analizar los llamados conceptos éticos densos, a saber, 
aquellos que tienen una faceta tanto descriptiva como valorativa como es el caso de "cruel" o "generoso". Éstos funcionan simultáneamente como juicios descriptivos y como juicios valorativos debido a que la facticidad y la valoración están entrelazadas. Por consiguiente, no se puede distinguir de modo tajante entre su contenido descriptivo y su componente valorativo.

Así pues la relación entre los hechos y los valores es holista. El conocimiento de los hechos presupone el conocimiento de los valores y viceversa. En efecto, siguiendo la influencia de James, Putnam considera que no existe ningún conjunto de hechos observables preestablecidos que pueda ser descrito, dado que los que consideremos como tales dependerán en parte de la cultura que sostengamos, que depende del lenguaje que utilicemos $(1992,32)$. Todos nuestros conocimientos están en función de nuestra forma de vida.

Por otro lado, en "La importancia del conocimiento no-científico" el autor analiza brevemente una de las defensas más populares a favor de la dicotomía hecho-valor que sostiene que para saber si hay valores necesitamos un órgano sensorial. Ésta versa del siguiente modo: “¿Cómo puede haber valores éticos objetivos? Podemos decir cómo detectamos amarillo, puesto que tenemos ojos, ¿pero qué órgano sensorial tenemos para detectar los valores?" (Putnam, 1997b, 4). Si uno examina dicho argumento puede observar que opera una noción ingenua de la percepción, que ignora el contenido conceptual involucrado en el proceso perceptivo, que se pone en evidencia cuando consideramos la siguiente pregunta planteada por Putnam: “Cómo podemos llegar a decir que las personas están alborozadas? Después de todo, no tenemos ningún órgano sensorial para detectar el alborozo" (1997b, 4). En este caso, se pone de manifiesto que podemos decir que otras personas están alborozadas, incluso ver que otras personas lo están, como los niños cuando reciben un regalo, porque previamente hemos adquirido el concepto de alborozo, porque soy capaz de interpretar la expresión lingüística en cuestión, de comprenderla. De esta manera, la estrategia putneana para socavar esta apelación a la imposibilidad de percibir valores consiste en dar un giro lingüístico y pragmático, que enfatiza la estrecha vinculación del lenguaje con la praxis. Dicho sea de paso esta maniobra es usada con frecuencia por el autor para explicitar su comprensión realismo de tipo natural.

Tal argumento en defensa de la dicotomía hecho-valor va acompañado de la idea de que la experiencia perceptiva es valorativamente neutral y que, en consecuencia, los valores son añadidos a la experiencia por proyección. Por el contrario, indica Putnam, la experiencia no es neutral, nos llega en medio de un griterío de valores. Ya que en la infancia experimentamos la comida y la bebida, los abrazos y la cordialidad como buenos, en cambio, la privación y el dolor como malos. Pero, en la medida en que las experiencias se multiplican y se convierten en más sofisticadas, los matices y las sombras de los valores se multiplican también y se convierten en más sofisticados, como lo muestra el caso de la descripción de un 
buen vino por parte de un catador (Putnam, 1997b, 4). Esta imagen muestra claramente la presencia del holismo entre hechos, valores e interpretaciones.

Sin embargo, queda una cuestión abierta: ¿cómo podemos distinguir entre lo objetivamente valioso y lo meramente valorado? La respuesta putneana es que solamente concluimos que algunas de las valoraciones están justificadas mientras que otras no lo están mediante la reflexión inteligente de nuestras valoraciones, lo que Dewey llama la "crítica". En otras palabras, el valor objetivo se desprende de la crítica de nuestras valoraciones. No obstante, la objetividad requerida por las afirmaciones éticas no es del tipo que proporciona una fundamentación platónica o de un género que esté ahí previamente "a nuestro entregarnos a la vida y reflexión ética”, sino que involucra la capacidad para superar la clase de crítica que emerge en las situaciones problemáticas con las que nos enfrentamos en la vida real, en las que "un marino calafatea su embarcación mientras aún está a flote" (Putnam, 2004,114). Volveré a estas cuestiones más adelante.

\section{Objetividad, lenguaje y razonabilidad}

La defensa de la objetividad en el ámbito del valor y de la experiencia humana, la creencia de que se puede afirmar que una concepción o forma de vida es mejor que la otra, por ejemplo, no exige sólo enfrentar a concepciones estrechas de la racionalidad y absolutas la objetividad, sino también batallar contra posiciones opuestas: las tendencias relativistas. Esta situación se expresa claramente en otras obras del autor, como es el caso de Cómo renovar la filosofía, en la cual declara su preocupación por la situación de la filosofía contemporánea, que estima que oscila entre la Escila del cientificismo y la Caribdis del relativismo, y en consecuencia, se encuentra en un estado de parálisis tal que requiere ser revitalizada, en otras palabras, la actividad de la filosofía necesita ser revisada para recuperar su sano curso (Putnam, 1994). Entre los representantes de las perspectivas relativistas que aparecen en escena figura Richard Rorty, quien afirma que deberíamos eliminar cualquier noción de mundo objetivo y hablar, en cambio, de los puntos de vista que "nuestra cultura" aceptaría. Putnam, rechaza su propuesta e insiste en seguir hablando de objetividad.

En efecto, explicita dos discursos en los que se utiliza el concepto, puesto que cree que la crudeza de las nociones de objetividad que a menudo operan en éstos es la fuente de las dificultades. En primer lugar, el de aquellos filósofos que intentan habitualmente definirla por medio de expresiones tales como "la realidad tiene una existencia y un carácter independiente de las prácticas, las creencias y las evidencias humanas" o "el que algo sea el caso es independiente de cómo lo consideraría cualquiera." En particular, para los realistas metafísicos contemporáneos el uso del término "objetivo" es pertinente sólo para afirmaciones que "realmente" tienen un valor de verdad. En segundo lugar, el modo de juzgar "objetivamente" cuando no hacemos metafísica, en el cual normalmente denominamos 
"subjetivos" a los enunciados que se hacen desde un punto de vista idiosincrático, por parte de personas que ignoran otros puntos de vista relevantes, mientras que denominamos "objetivos" a aquellos cuya pretensión de verdad no depende de los puntos de vista e intereses de los demás (Putnam, 1997b, 7).

No obstante, si tenemos en cuenta los discursos de la ética, en el cual las cuestiones morales están en función de las prácticas humanas, que involucran valoraciones y creencias filosóficas, religiosas y fácticas, cualquier pretensión de bosquejar una versión de la objetividad independiente del factor humano sería inútil. Ante este reconocimiento podemos optar por abandonar toda pretensión de objetividad, tal como sugiere Rorty, o bien, repensar y redefinir sus sentidos, buscando aquellos que hagan justicia a la praxis de los individuos. Esta segunda vía es la que elige Putnam, que procura abandonar la noción de objetividad asociada a la metáfora del ojo de dios, que involucra la idea de descripción correcta de los objetos y neutralidad valorativa. En La herencia del pragmatismo presenta, en su lugar, la comprensión de "objetividad humana", esto es, lo que es objetivo desde el punto de vista de nuestra mejor y más reflexiva práctica (1997a, 144).

Al dejar de equiparar la idea de objetividad con descripción se reconoce así la posibilidad de tomar en consideración enunciados que no implican necesariamente descripciones. De esta manera, hay enunciados que admiten términos como "correcto", "erróneo", "verdadero", "falso", "justificado" e "injustificado" que no son descripciones y, sin embargo, están bajo el control racional, son guiados por estándares apropiados a sus funciones y contextos particulares. La labor de describir el mundo, dice Putnam, es una función del lenguaje extremadamente importante, pero no la única. "Tampoco es la única a la que le son aplicables preguntas como: Este modo de realizar esta función ¿es razonable o no lo es? ¿Es racional o irracional? ¿Está justificado o no?” (2002, 49).

En consecuencia, los juicios de razonabilidad sí pueden ser objetivos, aunque esto no supone que sean totalmente independientes de lo que los seres humanos puedan saber y hacer, ni tampoco significa independencia de un contexto particular. Por eso, el autor propone que una condición suficiente (pero no necesaria) para que un juicio sea llamado objetivo es que sea razonable desde el punto de vista de un interés en el bienestar común, donde este bienestar no se considera como algo que viene dado, sino como que ha de determinarse mediante la discusión inteligente entre las personas que comparten este compromiso. Si al final de la discusión las personas implicadas aceptan una afirmación ética de esta clase, en la medida en que no surjan razones para cuestionarla, será lo que Dewey denomina "aseverables justificadamente" (Putnam, 1997b, 6-7). En otras palabras, lo objetivo es comprendido como aquello que es razonable aceptar en función del bienestar común, bienestar que se establece a partir del debate inteligente de las personas involucradas. Luego, si ellas aceptan una aseveración particular y no surgen razones que se opongan ésta será entonces una aseveración justificada. De este modo, la razón y la justificación son internas a una forma de la vida, donde 
"lo razonable" emana de la discusión inteligente de las personas que forman parte de ella.

\section{Pluralismo pragmático, normatividad y realismo natural}

El autor de Ética sin ontología arremete en dicha obra contra perspectivas éticas que intentan ofrecer una explicación ontológica de la objetividad de la misma, como la metafísica platónica y sus derivados contemporáneos. El ontólogo inflacionista, dice Putnam, pretende hablarnos de la existencia de cosas desconocidas para la percepción ordinaria y el sentido común, de cosas que son "invisibles", como en el caso de Platón, para quien la existencia de la forma del bien explica la existencia de los valores y las obligaciones éticas $(36,2013)$. De igual manera, a principios del siglo XX, en los Principia ethica de Moore se afirma que los juicios éticos tratan de una cualidad singular y supra sensible "el bien" y que decidimos la corrección de tales juicios mediante especial intuición de ese objeto suprasensible.

Estos enfoques pretenden fundamentar la objetividad del pensamiento ético en objetos, postulando algo "no natural", como entidades misteriosas o sublimes que permanecen invisible detrás la bondad de las personas, acciones o situaciones, y luego, intentan explicar por qué son buenas gracias a éstas. Asimismo, la metafísica deflacionista también juega sus cartas en este asunto. Ésta tiene dos vertientes, una reduccionista y otra eliminacionista. La primera afirma que tal cosa no es nada más que otra (que A no es nada más que B) como, por ejemplo, que "el bien no es nada más que placer", que "las propiedades no son nada más que nombres", que "las proferencias éticas no son nada más que sentimientos". La segunda, manifiesta que "tal propiedad u objeto no existe" y que nuestra conversación ordinaria sobre la ética es tan cognitivamente errónea como una sobre magia o alquimia. La exposición de estas perspectivas metafísicas y sus críticas tienen como finalidad, por un lado, hacer visible la pretensión de algunos filósofos de reducir todas las preguntas o los asuntos éticos y los problemas de valor a una única cuestión (por ejemplo, la presencia o la ausencia del bien), o bien, a eliminar toda carga cognitiva del discurso ético, es decir, sostener que las proposiciones de la ética hablan sobre cosas que no existen. Y por otro, mostrar que predomina una concepción de la objetividad de los juicios de valor ligada a la presencia de objetos, de entidades no naturales a partir de los cuales establecer la objetividad de los enunciados de valor ${ }^{1}$.

\footnotetext{
${ }^{1} \mathrm{El}$ autor considera que la filosofía heredó dos ideas de la metafísica platónica que aún persisten. A saber, en primer lugar, la idea de que si un enunciado es objetivamente verdadero, entonces, debe existir un objeto que se corresponda con el enunciado y, en segundo lugar, si éste no existe, entonces, deben existir objetos no naturales que desempeñen el papel de artífices de la verdad. Si aceptamos ambas ideas, dice Putnam, es muy probable que también se acepte que si un enunciado es verdadero, entonces, éste será una descripción de los objetos o las propiedades que lo convierten en verdadero $(2013,88)$.
} 
Ante estos proyectos metafísicos Putnam reacciona proponiendo como alternativa la tesis del pluralismo pragmático, que "no requiere descubrir objetos misteriosos y suprasensibles detrás de nuestros juegos de lenguaje” $(2013,42)$. Ésta puede ser comprendida en base a los juegos de lenguaje de Wittgenstein, desde un enfoque pragma-lingüístico, que implica el reconocimiento de que empleamos numerosos tipos diferentes de discursos en el lenguaje cotidiano, discursos sujetos a diferentes estándares, que poseen distintos tipos de aplicaciones, con diferentes rasgos lógicos y gramaticales, y que la verdad puede decirse dentro de los juegos de lenguaje que empleamos mientras el lenguaje opera. Gracias a esta tesis, Putnam puede dar cuenta de la normatividad presente en el plano del discurso ético, ya que los contextos lingüísticos son normativos, determinan las reglas de justificación y las condiciones de verdad de las aseveraciones. Además, tal como expresa Mayoral, este planteo evita el tener que ofrecer explicaciones en base a recursos de naturaleza ontológica y admite una pluralidad de discursos en las que se cifran nuestros modos de hablar de cuestiones científicas o de aspectos del sentido común (Mayoral, 2018, 9).

Aún más, como telón de fondo de tales cuestiones éticas se encuentra la adhesión del autor a un realismo de tipo natural, influenciada por el pragmatista James y su tendencia al realismo directo. A saber, en Sentido, sinsentido y todos los sentidos Putnam mantiene la tesis de que la percepción exitosa consiste en ver y escuchar las cosas que están ahí, de modo directo. Percibir es ver lo que hay $(2000,58)$. Por lo tanto, nuestra percepción no involucra interfaces, tales como instancias intermedias de representación de imágenes mentales que explican la conexión entre el sujeto y el mundo, sino que nos permite conectar directamente con el medio. Luego, nuestro lenguaje da cuenta de esta relación (Di Santo, 2017).

Este enfoque promueve así una actitud para abordar la relación del individuo y su entorno que reconoce como real las cosas que podemos experimentar directamente y cuyo conocimiento se obtiene a partir de la reflexión sobre nuestras prácticas lingüísticas, es decir, desde el vínculo del lenguaje con las prácticas. Por consiguiente, en este contexto filosófico hay una noción de verdad y de realismo que no son epistémicos, tal como señala Perona, que son las bases sobre la que se asientan las nociones epistémicas, pero no se agotan en ellas. Incluso, hay que destacar que el autor está desplazando y reformulando un problema clásico del realismo, que en su planteo tradicional consistía en garantizar la objetividad del conocimiento, y requiere antes determinar cómo nuestras percepciones del objeto se adecuan apropiadamente a dicho objeto, hacia un problema del lenguaje y la justificación, a saber, cómo saber que las condiciones de asertabilidad de un enunciado son suficientemente apropiadas (Perona, 2001, 295-297). 


\section{La objetividad y los límites de la justificación racional}

Como ya argumenté en otra ocasión, en el caso de las controversias éticas tales como la discusión sobre la legitimidad del aborto se manifiestan los límites de la justificación racional, debido a que se ponen en juego convicciones muy profundas de las personas. Desde luego, Putnam ejemplifica el desacuerdo ético con la disputa sobre la moralidad del aborto, que a veces se formula en términos metafísicos tales como ¿cuándo el feto se convierte en persona? ¿Cuándo el feto adquiere el alma? $(2013,122)$. En este caso, apelar al recurso de la justificación para ofrecer razones pertinentes por las cuales optamos una postura o una solución a un problema específico resulta inútil, nuestra pala se dobla, la justificación encuentra ahí su límite (Di Santo, 2019). En una lectura wittgensteniana tales cuestiones pertenecen al terreno de la certeza, que indican nada más que "un tono en el que se constata cómo son las cosas” (Wittgenstein, 2014b, \$30). De tal manera que ensayamos justificaciones a partir de nuestras convicciones, pero que se mueven en círculos.

Sin embargo, Putnam, frente a todos aquellos filósofos que apelan a que la presencia del desacuerdo es una razón suficiente para sostener que no hay verdad ni justificación en ética, insiste en salvar la razón y la justificación dentro de la vida ética ${ }^{2}$. Añade al respecto:

El enfoque correcto de nuestros problemas éticos no es ceder a la posibilidad misma de la discusión racional ni buscar un fundamento metafísico fuera de (o arriba de) todos los contextos y situaciones problemáticas, sino investigar, discutir e intentar acciones cooperativa y democráticamente, y sobre todo de manera falibilista. Lo terrible sobre la dicotomía hecho-valor es que, por negar que una discusión ética es responsable y racional, "bloquea el camino de la investigación desde el mismísimo inicio”. (Putnam, 2007, 187).

En realidad, la pregunta por los criterios para decidir quién tiene la razón cuando estamos ante desacuerdos éticos, para reconocer cuándo las aseveraciones están justificadas o cuándo es razonable aceptar algún juicio, no puede ser respondida apelando a un único criterio o método de decisión debido a la complejidad de las formas de vida, en las que se manifiesta la pluralidad y diversidad de prácticas humanas. Por tal razón, Putnam explicita que no precisamos un criterio único para juzgar la aseverabilidad justificada. En cambio, lo que necesitamos es, por un lado, el compromiso con la vida ética para decir "quiero ayudar" cuando

\footnotetext{
${ }^{2}$ Para mostrar que los desafíos éticos pueden ser superados, el autor argumenta que la ética del macho sólo fue socavada cuando un gran número de personas empezaron a "ver" que alguien que repudie entrar en ese juego no era necesariamente un pusilánime. Los grandes ejemplos morales del mundo, como Buda, Confucio, Sócrates, entre otros, demostraron en vida que podía existir gloria y dignidad al ponerse del lado de las víctimas y los oprimidos (Putnam, 2013, 54). Con ello, apela al conocimiento histórico y a un proceso reflexivo para combatir concepciones morales tales como el machismo.
} 
estoy frente al sufrimiento concreto de los otros y, por otro lado, acudir a la autoridad de la filosofía, que si bien no tiene ningún almacén privado de conocimientos o métodos para alcanzar la verdad ni algún acceso privado a lo bueno, siguiendo a Dewey, acepta las cosas buenas que están repartidas en la experiencia humana, pues posee la autoridad de la inteligente, de la crítica de los bienes comunes y naturales (Putnam, 2007, 186).

Los desafíos que ostentan los desacuerdos y problemas éticos ponen en evidencia la necesidad de buscar también a otros recursos, tales como recurrir a un compromiso de la voluntad para decir "aquí estoy" ante el sufrimiento del otro, una apertura hacia la ética de la compasión. Esta idea cobra mayor sentido dentro de una visión más amplia que el autor tiene de la filosofía, que no acaba en la actividad argumentativa, pues cree que "una filosofía compuesta únicamente por argumentaciones no puede satisfacer un hambre genuina (...)" (Putnam, 1992, 38) y, también, en el marco de una concepción holista del florecimiento humano según la cual el florecimiento de tipo intelectual es sólo una porción que tiene sentido en el marco de nuestro florecimiento humano en general.

Por otro lado, la referencia a la autoridad de la filosofía, a su capacidad crítica es constante en sus obras. En Ética sin ontología Putnam enfatiza un aspecto de la misma que llama "trascendencia reflexiva", que implica un ejercicio reflexivo del pensamiento para poner en tela de juicio no sólo las ideas recibidas, sino una crítica de nivel mayor en la cual se ponen en cuestión también los distintos modos en que estamos acostumbrados a pensar, una crítica de nuestros parámetros y modos de criticar $(2013,149)$. Esto muestra su fuerte confianza en que la filosofía nos posibilita, de alguna manera, "ver" o reconocer los aciertos y desaciertos tanto de nuestras ideas como de los parámetros con los cuales pensamos. Sin embargo, esta postura dispara algunas inquietudes, como por ejemplo, en vista a que los parámetros con los cuales juzga el filósofo también son normativos entonces ¿cómo prevenir que caigamos en una regresión al infinito? En clave wittgensteniana, si el filósofo puede tomar distancia su propio contexto moral y hacer una crítica de los parámetros con los que juzga ¿hay un nuevo juego desde el que está hablando el filósofo? ¿Hay un juego de lenguaje de la filosofía?

Por otra parte, abordar este problema epistemológico de los criterios para las aseveraciones éticas implica también un compromiso con una investigación inteligente de las situaciones problemáticas, que vale también para otras áreas del conocimiento. A saber, tal investigación reconoce, en primer lugar, que siempre nos valemos de un conjunto de valoraciones y descripciones que no se cuestionan en esa situación. En segundo lugar, tiene en cuenta, siguiendo a Peirce, que aprendemos a conducir la investigación examinando el éxito y el fracaso de los modos de conducir la investigación y, siguiendo a Dewey, que hemos aprendido que lo que es válido para la investigación en general lo es también para la investigación sobre los valores en particular. En tercer lugar, tiene que ser democrática y obedecer a los principios de la ética discursiva, que implican no bloquear los 
caminos de la investigación, evitar las relaciones de dependencia y jerarquía, e insistir en la experimentación mientras sea posible, sino en la observación y el análisis detallado (Putnam, 1997, 10-11). En este enfoque, los discursos adoptan ciertos preceptos que garantizan la participación y libertad de acceso, el derecho a cuestionar las aseveraciones y expresar las propias sin presión alguna, entre otros. Además, la importancia de proyectar la investigación en una sociedad democrática no es menor pues, tal como expresa Muñoz Olivera, una sociedad que se organiza por jerarquía limita la racionalidad de aquellos que se encuentran en los extremos pues esta jerarquía impide el desarrollo intelectual de los oprimidos y fuerza a los privilegiados a construir racionalizaciones sin sentido para justificar su postura (Muñoz Olivera, 2017, 238).

De igual manera, Putnam destaca que la distinción peirceana entre duda real y duda de papel arroja luz al problema de la objetividad moral debido a que pone de manifiesto que tanto la duda como la creencia requieren justificación (1997a, 145). A saber, Peirce sostiene que un paso preliminar a la investigación consiste en desechar ficciones, por eso advierte acerca de la duda de papel que mantienen algunos pensadores que proponen que la filosofía debe partir desde un estado mental. Uno de ellos sugiere que se comience dudando de todo, aunque mantiene que hay una sola cosa de la que no se puede dudar. Otro aduce que deberíamos comenzar observando las primeras impresiones del sentido, olvidando que las percepciones son nuestra elaboración cognitiva. A éstos Peirce les replica: "¿llama usted dudar al escribir en un pedazo de papel que usted duda? Si es así, la duda no tiene nada que ver con ningún quehacer serio" (1905, 161-181). Ciertamente, la duda auténtica es aquella que inicia la investigación, que está causada por la interacción con el mundo, que es involuntaria e incómoda ya que interrumpe la acción del pensamiento y nos estimula a indagar las creencias hasta destruirlas. Ésta sólo cesa cuando se obtiene la creencia. En efecto, esta distinción entre duda real y duda de papel indica la necesidad de contar con razones para dudar genuinamente.

En suma, la propuesta de perseguir una investigación inteligente, democrática y falibilista se presenta, a mi juicio, como una alternativa válida tanto para distinguir aseveraciones justificadas en los debates éticos como para sortear los escollos que producen algunos conflictos morales o controversias en la actualidad. Por ejemplo, el reconocimiento de la aseveración "la violencia de género es reprochable" o "pegar a una mujer está mal" como una aseveración justificada objetivamente se obtuvo gracias a diversos factores. Algunos de los cuales se reconocen en los desarrollos de Putnam, tales como los procesos de crítica de las creencias convencionales, instancias de intercambios de discursos, análisis de los sentidos del concepto violencia y resignificaciones, visibilización del problema por parte de miles de mujeres que mediantes diversas estrategias mostraron el sufrimiento que viven a diario otras mujeres y sensibilizaron a la comunidad. Luego, esto se reconoció como un problema específico y se insertó dentro de un proceso de 
investigación inteligente de conflictos éticos que, actualmente, forman parte de la agenda científica y de las políticas de estado de algunos países.

\section{Comentarios finales}

La propuesta de Putnam, si bien posee algunas oscuridades en su argumentación y deja abierta algunas cuestiones, representa un importante esfuerzo por poner a los asuntos de la ética en el terreno de la racionalidad y la objetividad. Una de las maniobras más destacadas del autor es analizar los sentidos disponibles del término "objetividad" y proponer otros que se ajusten al ámbito de la experiencia humana y su complejidad, al discurso del sentido común.

La objetividad que Putnam reconoce para las aseveraciones éticas no supone que ellas sean independientes de las prácticas humanas o de un contexto particular. Por eso, no exige neutralidad valorativa. En cambio, "objetivo" es aquello que es razonable aceptar en el contexto de una forma de vida, en función del bienestar común, bienestar que se establece a partir del debate inteligente de las personas involucradas. Ésta reivindica que no valen lo mismo todas las aseveraciones, que algunas están justificadas y otras no, y que los criterios que se necesitan para determinarlo implican procesos críticos y reflexivos, e instancias de debate que pongan en tela de juicio las valoraciones y las razones que mantenemos. Y si no se logra un acuerdo entre ellas, también disponemos de otros recursos para abordar las opiniones éticas controvertidas como la investigación inteligente.

\section{Referencias bibliográficas}

Bosso, Cristina (2018). Sobre los límites de la justificación. En Bosso, Cristina (Ed.), Wittgenstein. Mares del Lenguaje (pp.129-148). Córdoba: Lago Editora.

Di Santo, Laura B. (2017). Aspectos de la percepción y su relación con el realismo natural de Hilary Putnam. En Risco Mercedes y Stisman Andrés (Eds.), Lenguaje y conocimiento II (pp.59-72). Tucumán: Universidad Nacional de Tucumán.

Di Santo, Laura B. (2019). La ética pragmatista de Hilary Putnam. La objetividad y los límites de la justificación. En Rueda Leopoldo, Mattarollo Livio, Sánchez Victoria (Eds.), Actas del VI Coloquio Internacional de Filosofia del Conocimiento. Las buisquedas de la filosofía en la contemporaneidad. La actualidad del pragmatismo. La Plata: Universidad Nacional de La Plata.

Mayoral, Juan V. (2018). Hilary Putnam, la evolución del realismo y la perspectiva pragmatista. Análisis. Revista de investigación filosófica, 5(1), 3-25.

Muñoz Olivera, Luis (2017). La ética de Putnam contra el pluralismo inconmensurabilista. En Muñoz Olivera, Luis (Ed.), Aproximaciones a la diversidad cultural (pp.207-242). México: UNAM. 
Peirce, Charles (1905). Qué es el pragmatismo, The Monist, 15(2), 161-181. Traducción castellana de Norman Ahumada (2004), disponible en http://www.unav.es/gep/ WhatPragmatismIs.html

Perona, Ángeles (2001). ¿ Objetividad sin universalidad? El realismo de Hilary Putnam. En Arena Luis, Jacobo Muñoz, Perona Ángeles (Eds.), El retorno del pragmatismo (pp.289-309). Madrid: Trotta.

Putnam, Hilary (1992). El pragmatismo. Un debate abierto. Barcelona: Gedisa.

Putnam, Hilary (1994). Cómo renovar la filosofía. Madrid: Cátedra.

Putnam, Hilary (1997a). La herencia del pragmatismo. Barcelona: Ed. Paidós.

Putnam, Hilary (1997b). La importancia del conocimiento no-científico. Teorema, Suplemento Limbo, 2, 1-17.

Putnam, Hilary (2000). Sentido, sinsentido y todos los sentidos. Barcelona: Paidós.

Putnam, Hilary (2004). El desplome de la dicotomía hecho-valor y otros ensayos. Barcelona: Paidós.

Putnam, Hilary (2007). La ciencia y algunos filósofos. Solar, 3, 171-187.

Putnam, Hilary (2013). Etica sin ontología. Barcelona: Alpha Decay S.A.

Wittgenstein, Ludwig (1989). Conferencia sobre ética. Barcelona: Paidós.

Wittgenstein, Ludwig (2014a). Sobre la certeza. Barcelona: Gredos.

Wittgenstein, Ludwig (2014b). Investigaciones filosóficas. Barcelona: Gredos. 